\title{
Influência de fatores emocionais no desenvolvimento de doenças cardiovasculares: uma revisão narrativa
}

Influence of emotional factors in the development of cardiovascular diseases: a narrative review

Influencia de los factores emocionales en el desarrollo de enfermedades cardiovasculares: una

revisión narrativa

Layandra Vittória de Assis ${ }^{1 *}$, Amanda da Silva Dornelas ${ }^{2}$, Carolini Fernandes ${ }^{3}$, Carla Vitória de Araújo Macêdo ${ }^{4}$, João Pedro Vieira do Prado $^{5}$, Marcela Chiriano ${ }^{6}$, Márcia Luísa Monteiro Cunha ${ }^{7}$, Sarah Lopes Figueiredo ${ }^{8}$, Vivianne Araujo Rocha ${ }^{9}$, Guilherme Neif Vieira Musse ${ }^{10}$.

\section{RESUMO}

Objetivo: Analisar a fisiopatogenia e consequências patológicas do estresse frente a influência deste no desenvolvimento de doenças cardiovasculares, bem como sua prevenção e tratamento clínico. Revisão bibliográfica: As respostas fisiopatológicas do sistema cardiovascular relacionada a eventos de estresse desencadeiam uma complexa cascata imunológica que atingem diversas células do sistema imune, além de estar relacionado com a hiperatividade simpática e com a redução da atividade parassimpática. A prevenção das doenças cardiovasculares por meio da redução do estresse está relacionada a pelo menos três ocasiões: orientação a população sobre a importância da diminuição do estresse e seus efeitos adversos, estresse como fator de risco para grupos de alto risco e o estresse sendo considerado como um marcador de risco. $\mathrm{O}$ tratamento requer compreensão frente aos fatores de risco clínicos e psicológicos visando integrar tratamento médico, modificação do estilo de vida e manejo dos estressores psicossociais. Considerações finais: $O$ estresse afeta diretamente o sistema cardiovascular, portanto, práticas que tem demonstrado a redução tais sentimentos como mudanças de comportamento e no estilo de vida e modificação do fator de risco e bemestar psicossocial são importantes para aliviar o estresse e, consequentemente, o desenvolvimento de problemas cardíacos.

Palavras-chave: Doença das coronárias, Transtornos de ansiedade, Sistema cardiovascular, Estresse fisiológico.

\footnotetext{
ABSTRACT

Objective: To analyze the pathophysiology and pathological consequences in view of the influence related to emotional factors in the development of cardiovascular diseases as well as their prevention and clinical treatment. Bibliographic review: The pathophysiological responses of the cardiovascular system related to stress events trigger a complex immune cascade that affects several cells of the immune system, in addition to being related to sympathetic hyperactivity and the reduction of parasympathetic activity. The prevention of cardiovascular disease through stress reduction is related to at least three occasions: guidance to the population on the importance of reducing stress and its adverse effects, stress as a risk factor for high-risk groups and stress being considered as a risk marker. Treatment requires understanding of the clinical and

${ }^{1}$ Instituto Metropolitano de Ensino Superior do Vale do Aço (IMES UNIVAÇO), Ipatinga - MG.

*E-mail: layandraassis@yahoo.com.br

2Universidade Federal de Lavras (UFLA), Lavras - MG.

3Universidade Nove de Julho (UNINOVE), Guarulhos - SP.

${ }^{4}$ Universidade Federal de Pernambuco (UFPE), Recife- PE.

5 Universidade Nove de Julho (UNINOVE), Bauru - SP.

6 Universidade do Oeste Paulista (UNOESTE), Jaú - SP.

7 Universidade Salvador (UNIFACS), Salvador - BA.

¿Universidade de Itaúna (UIT), Itaúna - MG.

${ }^{9}$ Centro Universitário Tocantinense Presidente Antônio Carlos (UNITPAC), Araguaína - TO.

10Universidade Federal de Juiz de Fora (UFJF), Juiz de Fora - MG.
} 
psychological risk factors in order to integrate medical treatment, lifestyle modification and management of psychosocial stressors. Final considerations: Stress directly affects the cardiovascular system, therefore, practices that have been shown to reduce such feelings as changes in behavior and lifestyle, modification of the risk factor and psychosocial well-being are important to relieve stress and, consequently, the development of problems heart attacks.

Key words: Coronary disease, Anxiety disorders, Cardiovascular system, Physiological stress.

\section{RESUMEN}

Objetivo: Analizar la fisiopatología y las consecuencias patológicas ante la influencia relacionada con los factores emocionales en el desarrollo de las enfermedades cardiovasculares, así como su prevención y tratamiento clínico. Revisión bibliográfica: Las respuestas fisiopatológicas del sistema cardiovascular relacionadas con eventos de estrés desencadenan una compleja cascada inmune que afecta a varias células del sistema inmunológico, además de estar relacionada con la hiperactividad simpática y la reducción de la actividad parasimpática. La prevención de las enfermedades cardiovasculares mediante la reducción del estrés se relaciona con al menos tres ocasiones: orientación a la población sobre la importancia de reducir el estrés y sus efectos adversos, el estrés como factor de riesgo para los grupos de alto riesgo y la consideración del estrés un marcador de riesgo. El tratamiento requiere la comprensión de los factores de riesgo clínicos y psicológicos para integrar el tratamiento médico, la modificación del estilo de vida y el manejo de los factores de estrés psicosociales. Consideraciones finales: El estrés afecta directamente al sistema cardiovascular, por lo que prácticas que han demostrado reducir sentimientos como cambios en el comportamiento y estilo de vida, modificación del factor de riesgo y bienestar psicosocial son importantes para aliviar el estrés y, en consecuencia, el desarrollo de problemas ataques al corazón.

Palabras clave: Enfermedad coronaria, Trastornos de ansiedad, Sistema cardiovascular, Estrés fisiológico.

\section{INTRODUÇÃO}

O estresse é uma condição fisiológica que permite ao indivíduo reagir a diversos contextos que demandam esforço ou adaptação além de sua resposta basal. A visão do estresse aplicada à resposta de luta ou fuga permitiu a perpetuação da espécie humana, permanecendo como uma característica inerente à evolução adaptativa (RUSSELL G e LIGHTMAN S, 2019).

Entretanto, quando essas reações são exacerbadas ou prolongadas, podem causar excitação emocional e desencadear um desequilíbrio da homeostasia, além de disparar um processo de manifestações sistêmicas como distúrbios fisiológicos e psicológicos (MARGIS R, et al., 2003).

Recentemente, vários estudos epidemiológicos prospectivos e estudos científicos estabeleceram a associação entre doença cardiovascular e várias condições psicológicas, incluindo depressão, estresse emocional e ansiedade. Estes fatores emocionais estão muito presentes na sociedade contemporânea devido a transformação do estilo de vida e as relações sociais que contribuem para o acentuado estresse emocional, que repercutem em vários problemas de saúde mental e física, por meio de alterações do sistema endócrino, cardiovascular, imunológico e cerebral (KOMORI T, et al., 2019).

O sistema cardiovascular tem papel importante na modulação e manutenção de parâmetros como pressão arterial e frequência cardíaca evocadas em situações de estresse emocional, a fim de fornecer suporte hemodinâmico e metabólico adequado ao indivíduo sob condições fisiológicas que demandam mais esforço físico. Contudo vários estudos já estabeleceram possíveis mecanismos pelos quais o estresse exacerbado e os transtornos emocionais a longo prazo podem desencadear alterações fisiopatológicas no coração e na vasculatura e, portanto, ser fator de risco para várias patologias cardíacas (FIORANELLI M, et al., 2018; WIRTZ PH e VON KÄNEL R, 2017; CRESTANI CC, 2016).

De acordo com Shimokhina NY (2020), a prevalência de transtornos ansiosos e depressivos (TAD) em pacientes com patologias cardiovasculares varia de $20 \%$ a $45 \%$. Ademais, é notável que o risco de mortalidade cardiovascular em pacientes com síndrome coronariana aguda (SCA) e TAD foi de 2 a 2,6 vezes maior do que em pacientes sem transtornos emocionais (HERT M, et al., 2018). 
Ainda assim, alguns estudos já evidenciaram a relação entre os fatores emocionais e doenças cardiovasculares por meio de diversas vias de mediações plausíveis, em que fatores comportamentais, ativação do eixo hipotálamo-hipófise-adrenal (HPA), desregulação autonômica, respostas inflamatórias, disfunção endotelial e ativação plaquetária estão entre as mais importantes discutidas. Essas modificações fisiopatológicas induzidas pelo estresse resultante da ativação dessas vias incluem hipertensão, inflamação vascular e aumento da coagulação, alterações que, se repetidas ou persistentes, eventualmente levam a danos vasculares permanentes e aterosclerose. Essas disfunções, quando somadas a alguma doença de base cardíaca, desencadeiam mais precocemente os sintomas ou resultam em doenças mais graves $(\mathrm{OCH}$ DAG, et al., 2020).

Outro ponto relevante é a relação entre o estresse e o infarto agudo do miocárdio (IAM). Um estudo realizado com 11.119 casos e 13.648 controles em 52 países, intitulado Intercontinental Interheart, constatou que a presença de estressores dobra o risco de IAM (ROSENGREN A, et al., 2004).

Esses dados foram posteriormente confirmados por um inquérito nacional de saúde americano (FERKETICH AK, et al., 2005). No entanto, mesmo com tais evidências, cabe ainda investigar de maneira mais específica como esses fatores emocionais contribuem para o aumento do risco cardíaco e, consequentemente, o expresso número de morbidade e mortalidade nessa população.

Desse modo, o presente estudo tem como objetivo analisar a interferência e as consequências do estresse crônico e de origem emocional nas faces da cardiologia. Além disso, visa divulgar a importância do controle emocional e os entraves que o desequilíbrio do mesmo pode promover ao indivíduo afetando diretamente a sua qualidade de vida.

\section{REVISÃO BIBLIOGRÁFICA}

\section{Aspectos gerais dos fatores emocionais}

As mudanças sociais e demográficas na sociedade moderna se refletem na prevalência do estresse mental entre os indivíduos e, como resultado, o estresse relacionado ao trabalho, a solidão e isolamento social têm sido as principais fontes de distúrbios emocionais em humanos. Esse cenário tão frequente no corpo social é uma condição que desencadeia no indivíduo disfunções psicossociais, como depressão, ansiedade e estresse emocional, além de contribuir para alterações físicas de diversos sistemas como: endócrino, cardiovascular e cerebral (LI H e XIA N, 2020; KOMORI T, et al., 2019).

Os fatores estressores influenciam diretamente nos transtornos emocionais, gerando um agravamento de diversas doenças a longo prazo que resultam em um problema de saúde pública. A maioria das pessoas não consegue lidar com suas fontes de tensão, o que reflete no aumento da prevalência do estresse. Dados da cidade de São Paulo, maior cidade da América Latina, apontam que no ano de 2004 40\% de seus habitantes estavam expostos constantemente ao estresse (LIPP MEN, 2004 apud SADIR AM, et al., 2010).

Logo, entre os fatores estressores, os mais comuns são a depressão, ansiedade e o estresse propriamente dito. A depressão pode ser classificada como um transtorno de humor, regendo as atitudes dos sujeitos modificando a percepção de si mesmos, passando a enxergar seus entraves como grandes catástrofes (ESTEVES F e GALVAN A, 2006).

Já a ansiedade é definida como um sentimento vago e desagradável de medo e apreensão, caracterizado por tensão ou desconforto derivado de antecipação de perigo, de algo desconhecido ou estranho (CASTILHO A, et al., 2000). Por fim, pode-se classificar o estresse como uma reação do organismo que ocorre frente a situações que exijam dele adaptações além do seu limite (SADIR MA, et al., 2010).

Dessa forma, os fatores estressores podem causar alterações sistêmicas, como foi evidenciado por Tawakol A, et al. (2017), em um estudo longitudinal realizado com 239 pacientes, com idade média de 55 anos. Os pesquisadores concluíram que a atividade da amígdala, uma região do cérebro envolvida no estresse, também está envolvida parcialmente em doenças cardiovasculares por meio de um caminho que inclui aumento da atividade da medula óssea e inflamação arterial. 


\section{Mecanismos de adaptação do sistema cardiovascular frente a agravos}

Os organismos multicelulares se adaptam a situações de estresse através de complexas alterações neurais, humorais e celulares que envolvem múltiplos órgãos e tecidos. Esse sistema de adaptação é constituído por componentes do sistema nervoso central, incluindo neurônios do núcleo paraventricular do hipotálamo, núcleos adrenérgicos do tronco cerebral com componentes periféricos, o eixo hipotálamohipófise-adrenal e o sistema nervoso autônomo. Este sistema exerce importante influência em muitas funções vitais relacionadas a respiração, tônus cardiovascular e metabolismo, que também são alterados quando somos expostos a situações de estresse (LOURES DL, et al., 2002).

A importância do sistema endócrino para regulação da homeostase foi descoberta em 1930 em um estudo de Hans Seyle, quando o sistema simpático e o eixo hipotálamo-hipófise-adrenal foram descritos em resposta a uma lesão física, esforço e também estresse emocional. No meio do século passado foram detectados pela última vez nos átrios cardíacos, fragmentos similares aos das células endócrinas. No ano de 1970 foi provada a capacidade desses fragmentos na redução da pressão sanguínea através do seu efeito diurético e natriurético. De acordo com pesquisas recentes, o coração pode responder a uma lesão tecidual através de uma complexa cascata inflamatória, agindo com um "órgão do sistema imunológico". Diversas células como macrófagos, células dendríticas, mastócitos e linfócitos B e T estão presentes no coração. A partir disso, percebe-se a relação entre o sistema cardiovascular, as respostas fisiopatológicas ao estresse e como isso pode desequilibrar a homeostase do organismo e trazer patologias ao sistema cardiovascular (FIORANELLI M, 2018; RUSSELL G e LIGHTMAN S, 2019).

\section{Fisiopatologia das consequências do estresse na cardiologia}

A fisiopatologia envolvida no processo de estresse com afecções cardiológicas se dá através da modulação da atividade simpática e atuação do sistema renina-angiotensina-aldosterona. Este sistema atua de forma que o peptídeo Angiotensina II se envolve na interação com receptores vasculares do subtipo AT1, que, quando em atividades de estresse, mostram-se com níveis elevados junto da atividade plasmática (DOS SANTOS RAS, 2000).

A Angiotensina II, quando em níveis alterados, causa uma cascata de efeitos deletérios para o sistema cardiovascular, como o aumento da resistência vascular, hipertensão, hipertrofia de miócitos, efeito trófico, aumento do inotropismo e cronotropismo, estímulo à produção de radicais livres e substâncias próinflamatórias, resultando, assim, em uma remodelação cardiovascular (TRAPP SM, 2009).

Em contraponto à resposta da Angiotensina II, a Aldosterona estará reduzida pela inibição da enzima aldosterona sintase e seu efeito a longo prazo, quando não inibido, encontra-se também envolvido no processo de hipertrofia cardíaca, pelo aumento da síntese de colágeno tipo I e acúmulo de proteínas, podendo levar à falência do órgão. Frente a um cenário de estresse, outro grande sistema que também sofre alterações junto ao cardíaco é o metabólico, visto que, de acordo com Loures DL (2002), observa-se aumento da resistência à insulina, níveis elevados de glicemia e lipólise em indivíduos submetidos a testes. Tais efeitos são ocasionados pelo aumento da atividade adrenérgica, que é mediada pela ação das catecolaminas adrenalina (epinefrina) e noradrenalina (norepinefrina).

Quando se fala de estresse, é importante mencionar que o sistema cardiovascular atua ativamente diante dessas situações, alterando a frequência cardíaca, aumentando a pressão arterial e, consequentemente, o débito cardíaco. Em consenso com estudos recentes, o estresse agudo, demonstrado pelo aumento nos níveis séricos de serotonina em ratos, pode levar a um aumento da ativação plaquetária, aumento da viscosidade sanguínea e reduções drásticas do volume circulante plasmático, contribuindo para possíveis afecções cardíacas como aterosclerose e infarto agudo do miocárdio (IAM) (LOURES DL, et al., 2002).

O IAM, abordado como uma das consequências patológicas do estresse, é classificado como a morte dos cardiomiócitos por um processo de isquemia prolongada, desencadeada por ruptura de um vaso e consequente oclusão súbita por formação de trombo sobre placas ateroscleróticas em processo de inflamação. Se houver a obstrução de uma das artérias coronárias que irriga o coração, tal processo resulta em sucessivas lesões por distúrbios eletrolíticos, alterações morfológicas reversíveis e, por fim, danos 
definitivos precedidos de necrose do tecido em questão pela falta de oxigenação. O IAM ocasionado por estresse e maus hábitos está entre as principais causas de morte súbita no Brasil, o que mostra a importância do acompanhamento médico em sua prevenção e tratamento (PESARO AEP, et al., 2004).

Outra consequência grave ocasionada pelo estresse, que também já foi abordada nesse artigo, é a aterosclerose. Essa doença é caracterizada pelo agrupamento de monócitos dentro das paredes das artérias, onde essas células passam a acumular lipídeos, provocando um espessamento daquele local e formação de placas de revestimento no interior desses vasos. Com o passar do tempo e a contínua diminuição da luz do vaso, a passagem do sangue torna-se cada vez mais difícil, ocasionando pouca ou nenhuma oxigenação dos tecidos irrigados por esses vasos. Os primeiros sintomas da aterosclerose incluem o cansaço e cianose periférica, seguidos de cãibras depois da realização de pequenos esforços. Quando a obstrução está presente em um dos vasos do coração, ocorre o sinal clássico de Angina, indicando a falta de oxigenação do músculo cardíaco. Se não tratada a tempo, aterosclerose pode levar à síndrome coronariana aguda (infarto) e à morte (PORTAL VL, 2004).

Diante do exposto, a associação do estresse mental com eventos cardio-patológicos relaciona-se com a hiperatividade simpática e uma redução da atividade parassimpática. A hiperatividade simpática atua diretamente no aumento da frequência cardíaca, aumento da força de contração e fluxo sanguíneo. Essas alterações favorecem a instabilidade elétrica miocárdica, levando ao desenvolvimento de arritmias ventriculares letais e lesão endotelial, estimulando o sistema de reparo que dificulta o débito cardíaco devido a substituição de tecidos especializados (FIORANELLI M, 2018).

De acordo com um levantamento de dados publicado pela Nature Reviews Cardiology, situações de desgaste mental afetam também o sistema imunológico, provocando uma inflamação crônica e aumentando a produção de leucócitos que se acumulam nas paredes das artérias, diminuindo a luz do vaso e consequentemente dificultando a passagem do sangue, o que leva ao risco de formação de coágulo e obstrução do vaso, que pode culminar, futuramente, em infarto (KIVIMAKI M e STEPTOE A, 2018).

\section{Prevenção de doenças cardiovasculares por meio da redução do estresse}

A importância do estresse como alvo da prevenção das doenças cardiovasculares, universal ou direcionada, permanece sendo objeto de debate. Nesse contexto, existem pelo menos três conjunturas para tal prevenção. Primeiro, o estresse considerado como um fator de risco universal: esta estratégia é a alternativa de maior alcance, instigando toda a população a diminuir o estresse ou os seus efeitos adversos. Segundo, o estresse titulado como um fator de ameaça em grupos de alto risco: esta opção assume que a associação entre estresse e doenças cardiovasculares é causal, mas apenas as intervenções antiestresse orientadas são clinicamente significativas e rentáveis. Terceiro, o estresse sendo considerado como um marcador de perigo: neste contexto, o estresse é tratado como um indicador de risco para reconhecer grupos que precisem de prevenção orientada que se concentra na gestão de fatores de risco cardiovasculares padrão neste grupo (MACLEOD J e DAVEY SG, 2003).

Para os fatores de estresse difíceis de manejar, como o que ocorre em grupos que possuem alto risco para desenvolvimento de doenças cardiovasculares e que passaram por estresse emocional devido a catástrofes naturais ou a situações adversas (a exemplo, perda de parente próximo e desemprego), uma forma de progredir, além da busca de ajuda psicológica, é intervir sobre os fatores intermediários, como o sedentarismo, a alimentação desregulada e a obesidade. Como a doença cardiovascular é uma condição multifatorial, a retirada de qualquer dos fatores de risco padrão reduziria o risco global (ROZANSKI A, 2012). A atividade física, por exemplo, tem sido sugerida como uma intervenção para mitigar os efeitos adversos do estresse. Estudos demonstram que a prática de exercícios físicos pode reduzir os sentimentos de angústia, tais como depressão e ansiedade, além de reduzir os riscos de doenças cardiovasculares, exercendo um efeito protetor sobre tais patologias (ANDERSON L, et al., 2016; REBAR AL, et al., 2015; LEAR SA, et al., 2017).

Os programas de bem-estar no local de trabalho são concebidos para dar apoio a estilos de vida saudáveis e isso inclui exames de saúde de rotina, facilitação a uma alimentação equilibrada, gestão de peso e atividade física, sendo benéficos para pessoas que sofrem com o estresse (FONAREW GC, et al., 2015). 
Além disso, reabilitação cardíaca abrangente para pacientes que possuem doenças cardiovasculares inclui um componente psicológico relacionado com a mudança de comportamento no estilo de vida, modificação do fator de risco, e bem-estar psicossocial. Dessa forma, é evidente que tais mudanças são importantes para mitigar o estresse e, consequentemente, a possibilidade de desenvolvimento de problemas cardíacos advindos do esgotamento emocional (POGOSOVA N, et al., 2015).

\section{Tratamento clínico}

O tratamento das doenças cardiovasculares requer a compreensão dos fatores de risco clínicos e psicológicos que influenciam no seu surgimento e desenvolvimento, com o objetivo de conscientizar as pessoas sobre a importância de um estilo de vida saudável e maior proteção cardiovascular. Os fatores psicossociais são reconhecidos pelos pacientes como uma das importantes causas do seu adoecimento, ressaltando-se vivências de perdas e sobrecarga laboral (KNEBEL IL e MARIN AH, 2018).

Além disso, o estresse afeta diretamente a saúde física. Como essa é uma doença psicossomática, comumente a forma de tratamento é principalmente medicamentosa e atrelado ao aparecimento de sintomas físicos os fármacos utilizados frequentemente são antidepressivos e ansiolíticos, que possuem, como consequência, danos ao corpo humano e até chega a gerar dependência. Atualmente, nota-se uma preocupação mais acentuada das pessoas acerca da saúde física e mental. Então percebe-se um aumento da procura por tratamentos não medicamentosos, menos invasivos e prejudiciais ao organismo. Porém, ainda há bastante dúvidas acerca desses tratamentos sobre sua eficácia e eficiência por não terem estudos suficientes para comprovação científica (SILVA LC e SALLES TLA, 2016).

Pesquisas indicam o Treino do Controle de Stress (TCS) e a Terapia cognitivo comportamental (TCC) como eficazes no tratamento da ansiedade e depressão, bem como na modulação da reatividade cardiovascular em momentos de estresse emocional. Estas condutas auxiliam tanto na adesão ao tratamento quanto na reestruturação do modo de pensar, conduzindo a uma mudança estável e duradoura do estilo de vida, mais compatível com o autocuidado necessário no manejo de doenças crônicas. A abordagem cognitiva comportamental está sendo evidenciada, através de ensaios randomizados e metanálises, como eficaz no tratamento da depressão e da ansiedade (QUINTANA JF, 2011).

Além disso, uma abordagem terapêutica deve ser direcionada às vias pelas quais um gatilho de estresse mental agudo pode resultar em um agravamento de um fator patológico cardíaco como, por exemplo, a prevenção de uma possível ruptura de placa de aterosclerose por um episódio de estresse mental que posteriormente pode resultar em eventos cardiovasculares catastróficos, como um IAM. Dessa forma, já que o estresse emocional afeta o sistema cardiovascular aumentando o tônus simpático, alterando o estresse de cisalhamento e a coagulação, é de suma importância prescrever terapias farmacológicas que visem essas vias a fim de impedir que esse gatilho mental cause a ruptura da placa, trombose intraluminal e sequelas cardiovasculares (SCHWARTZ BG, et al., 2012). Logo, destaca-se que uma intervenção eficaz deve agregar tratamento médico, modificações no estilo de vida e manejo dos estressores psicossociais.

\section{CONSIDERAÇÕES FINAIS}

O estresse exerce papel importante para o ser humano deixando-o alerta para situações de luta ou fuga, sendo tal comportamento essencial para evolução da espécie humana. Logo, não se deve ter uma perspectiva tão negativa frente a ele, visto que o detentor desse papel negativo é a resposta em demasia. Dessa maneira, deve-se combater o estresse em excesso, não a resposta fisiológica. Sabe-se, ainda, que o órgão cardíaco necessita de condições favoráveis para o seu bom funcionamento, contudo, o estresse afeta diretamente 0 sistema cardiovascular gerando, por exemplo, o advento do contínuo estímulo de hormônios como serotonina, cujas conjunções são diretamente afetadas e exigem mais do coração. Portanto, diante da sucessiva dinamicidade do estilo de vida da maioria da sociedade, o estresse faz-se presente no cotidiano de muitos e, devido a isso, essa parcela já enfrenta os efeitos nocivos desse comportamento excedente. Para combater as consequências nocivas do estresse a longo ou a curto prazo, recomenda-se a prática de atividades físicas, intentar uma alimentação saudável e balanceada, junto à profissionais capacitados com objetivo de proporcionar melhor qualidade de vida. 


\section{REFERÊNCIAS}

1. ANDERSON L, et al. Exercise-based cardiac rehabilitation for coronary heart disease: Cochrane systematic review and meta-analysis. Journal of the American College of Cardiology. 2016; 67(1): 1-12.

2. CASTILLO A, et al. Transtornos de ansiedade. Rev. Bras. Psiquiatr., 2000; 20-23.

3. CRESTANI CC. Emotional stress and cardiovascular complications in animal models: a review of the influence of stress type. Frontiers in physiology, 2016; 251.

4. DOS SANTOS RAS, et al. Efeitos cardiovasculares e renais do sistema renina-angiotensina. Rev Bras Hipertens, 2000; 227-36.

5. ESTEVES FC, GALVAN AL. Depressão numa contextualização contemporânea. Aletheia, Canoas, 2006 ; $127-135$.

6. FERKETICH AK, BINKLEY PF. Psychological distress and cardiovascular disease: results from the 2002 National Health Interview Survey. Eur Heart J. 2005; 26(18): 1923-9.

7. FIORANELLI M, et al. Stress and inflammation in coronary artery disease: a review psycho neuroendocrine immunology based. Frontiers in immunology, 2018; 2031.

8. FONAROW GC, et al. Workplace wellness recognition for optimizing workplace health: a presidential advisory from the American Heart Association. Circulation, 2015; 131(20): 480-497.

9. HERT M, et al. The intriguing relationship between coronary heart disease and mental disorders. Dialogues in clinical neuroscience, 2018; 31.

10. KIVIMAKI, M., STEPTOE, A. Effects of stress on the development and progression of cardiovascular disease. Nat Rev Cardiol, 2018; 15, 215-229.

11. KNEBEL IL, MARIN AH. Fatores psicossociais associados à doença cardíaca e manejo clínico psicológico: percepção de psicólogos e paciente. Rev. SBPH [online]. 2018; 112-131.

12. KOMORI T, et al. Social status and modern-type depression: A review. Brain and behavior, 2019.

13. LEAR SA, et al. The effect of physical activity on mortality and cardiovascular disease in 130000 people from 17 high-income, middle-income, and low-income countries: the PURE study, 2017; 10113(390): 2643-2654.

14. LI H, XIA N. The role of oxidative stress in cardiovascular disease caused by social isolation and loneliness. Redox Biology, 2020.

15. LOURES DL, et al. Estresse Mental e Sistema Cardiovascular. Arquivos brasileiros de cardiologia, 2002.

16. MACLEOD J, DAVEY SG. Psychosocial factors and public health: a suitable case for treatment. Journal of Epidemiology and Community Health, 2003; 57(8): 565-570.

17. MARGIS R, et al. Relação entre estressores, estresse e ansiedade. Revista de Psiquiatria do Rio Grande do Sul,2003; 65-74.

18. OCH DAG, et al. Negative emotional states and negative life events: Consequences for cardiovascular health in a general population. Journal of Psychosomatic Research, 2020.

19. PESARO AEP, et al. Infarto Agudo do Miocárdio - Síndrome Coronariana Aguda com supradesnível do segmento ST. São Paulo, SP. Rev Assoc Med Bras, 2004.

20. POGOSOVA N, et al. Psychosocial aspects in cardiac rehabilitation: from theory to practice. A position paper from the Cardiac Rehabilitation Section of the European Association of Cardiovascular Prevention and Rehabilitation of the European Society of Cardiology. European Journal of Preventive Cardiology. 2015; 22(10): 1290-1306.

21. PORTAL VL. Aterosclerose - uma doença complexa. Revista da Sociedade de Cardiologia do Rio Grande do Sul, 2004.

22. QUINTANA JF. A relação entre hipertensão com outros fatores de risco para doenças cardiovasculares e tratamento pela psicoterapia cognitivo comportamental. Rev. SBPH [online]. 2011; 03-17.

23. REBAR AL, et al. A meta-meta-analysis of the effect of physical activity on depression and anxiety in non-clinical adult populations. Health Psychology Rev. 2015; 9(3): 366-378.

24. ROSENGREN A, et al. Association of psychosocial risk factors with risk of acute myocardial infarction in 11.119 cases and 13.648 controls from 52 countries (The INTERHEART Study): case-control study. Lancet. 2004; 364(9438): 953-62.

25. ROZANSKI A. Exercise as medical treatment for depression. J. Am. Coll. Cardiology, 2012; 60(12): 1064-1066.

26. RUSSELL G, LIGHTMAN S. The human stress response. Nature Reviews Endocrinology, 2019; $15(9): 525-534$.

27. SADIR MA, et al. Stress e qualidade de vida: influência de algumas variáveis pessoais. Paidéia (Ribeirão Preto), Ribeirão Preto, 2010; 73-81.

28. SCHMIDT K, et al. Um Olhar sobre o Stress nas Mulheres com Infarto Agudo do Miocárdio. Arq. Bras. Cardiol, 2020; 649-657.

29. SILVA LC, SALLES TLA. O estresse ocupacional e as formas alternativas de tratamento. Revista de Carreiras e Pessoas. 2016; 234-247.

30. SHIMOKHINA NY, et al. Peculiarities of Platelet Metabolism in Patients with Acute Coronary Syndrome with AnxietyDepressive Disorders and Informativity of Enzymes in the Forecast of Development of Cardiovascular Complications. Pharmaceuticals, 2020; 169.

31. TAWAKOL A, et al. Relation between resting amygdalar activity and cardiovascular events: a longitudinal and cohort study, 2017; 834-845.

32. TRAPP SM, et al. Efeitos da Angiotensina II no sistema cardiovascular. Archives of Veterinary Science, 2009; 233243.

33. WIRTZ PH, VON KR. Psychological stress, inflammation, and coronary heart disease. Current cardiology reports, $2017 ; 111$. 